PROCEEDINGS OF THE

AMERICAN MATHEMATICAL SOCIETY

Volume 127, Number 4, April 1999, Pages 1089-1096

S 0002-9939(99)04649-3

\title{
GAUSSIAN ESTIMATES AND REGULARIZED GROUPS
}

\author{
QUAN ZHENG AND JIZHOU ZHANG
}

(Communicated by Palle E. T. Jorgensen)

\begin{abstract}
We show that if a bounded analytic semigroup $\{T(z)\}_{\operatorname{Re} z>0}$ on $L^{2}(\boldsymbol{\Omega})\left(\boldsymbol{\Omega} \subset \mathbf{R}^{n}\right)$ satisfies a Gaussian estimate of order $m$ and $A_{p}$ is the generator of its consistent semigroup on $L^{p}(\boldsymbol{\Omega})(1 \leq p<\infty)$, then $i A_{p}$ generates a $\left(1-A_{p}\right)^{-\alpha}$-regularized group on $L^{p}(\boldsymbol{\Omega})$ where $\alpha>2 n\left|\frac{1}{2}-\frac{1}{p}\right|$. We obtain the estimate of $\left(\lambda-A_{p}\right)^{-1}(|\arg \lambda|<\pi)$ and the $p$-independence of $\sigma\left(A_{p}\right)$, and give applications to Schrödinger operators and elliptic operators of higher order.
\end{abstract}

\section{INTRODUCTION}

Let $\boldsymbol{\Omega}$ be an open set of $\mathbf{R}^{n}$ and let $T_{p}=\left\{T_{p}(t)\right\}_{t \geq 0}$ be the consistent semigroup on $L^{p}(\boldsymbol{\Omega})(1 \leq p<\infty)$ with generator $A_{p}$. It is known that the Gaussian estimate of $T_{p}$ is related to the $p$-independence of the spectrum $\sigma\left(A_{p}\right)$. For example, Arendt [2] proved that $\sigma\left(A_{p}\right)$ is independent of $p \in[1, \infty)$ when $A_{2}$ is self-adjoint and $T_{2}$ has a Gaussian estimate of order 2. Another important work in this direction is due to Davies [6]. On the other hand, the analyticity of $T_{p}$ was recently studied by some authors. Ouhabaz [17] showed that $T_{p}$ is an analytic semigroup of angle $\frac{\pi}{2}$ if $T_{2}$ is a self-adjoint semigroup satisfying a Gaussian estimate of order 2. Ouhabaz's result was generalized to a more general case by Hieber [12]. That is, if $T_{p_{0}}$ is an analytic semigroup of angle $\phi \in\left(0, \frac{\pi}{2}\right]$ and has a Gaussian estimate of order $m$ for some $p_{0} \in(1, \infty)$, so is $T_{p}$. In particular, he obtained the kernel estimates of $T_{p}(z)$ $\left(|\arg z|<\phi^{\prime}\right)$ and $\left(\lambda-A_{p}\right)^{-1}\left(|\arg \lambda|<\phi^{\prime}+\frac{\pi}{2}\right)$, where $\phi^{\prime} \in(0, \phi)$.

Also, we look at the Schrödinger operator $i(\Delta-V)$ with some potential $V$. It is Hörmander [14] who showed that the Schrödinger operator, even for $V \equiv 0$, generates a strongly continuous semigroup (i.e., $C_{0}$-semigroup) on $L^{p}\left(\mathbf{R}^{n}\right)$ if and only if $p=2$. Starting from this point, Pang [18] first applied regularized semigroups to $i(\Delta-V)$, and his result was improved by Boyadzhiev and deLaubenfels [4] in the language of regularized groups. It is worth noticing that, in [4], the problem was approached by using the boundary values of analytic semigroups.

The purpose of this paper is to extend all these results to more general situations. In section 2, we show the estimates of $T_{p}$ and its kernel on the right halfplane, and then deduce that $i A$ generates a regularized group on $L^{p}(\boldsymbol{\Omega})$ with suitable

Received by the editors February 27, 1997 and, in revised form, July 14, 1997 and July 22, 1997.

1991 Mathematics Subject Classification. Primary 47D03, 47F05.

Key words and phrases. Gaussian estimate, regularized group, analytic semigroup, differential operator.

This project was supported by the National Science Foundation of China.

(C)1999 American Mathematical Society 
regularizing operator. In section 3 , we obtain the estimates of $\left(\lambda-A_{p}\right)^{-1}$ and its kernel on the region $|\arg \lambda|<\pi$, as well as the $p$-independence of spectrum $\sigma\left(A_{p}\right)$. Finally, the section 4 is concerned with applications to Schrödinger operators and elliptic operators of higher order.

\section{Preliminaries}

Throughout this paper, for a linear operator $A$ on a Banach space $X$, we will denote by $\mathcal{D}(A)$ its domain, $\sigma(A)$ its spectrum, $\rho(A)$ its resolvent set, and $R(\lambda, A)$ its resolvent. Let $\Omega \subset \mathbf{R}^{n}$ be an open set. The Banach space of all bounded linear operators from $L^{p}(\boldsymbol{\Omega})$ to $L^{q}(\boldsymbol{\Omega})$ will be denoted by $B\left(L^{p}(\boldsymbol{\Omega}), L^{q}(\boldsymbol{\Omega})\right)$ with norm $\|\cdot\|_{q, p}(1 \leq p, q \leq \infty)$. Moreover, set $\Delta_{\theta}=\{z \in \mathbf{C} \backslash\{0\} ;|\arg z|<\theta\}$ for $\theta \in(0, \pi]$.

We start with the definitions of analytic semigroups and regularized groups (cf. $[7,19])$.

Definition 1.1. (a) Let $\theta \in\left(0, \frac{\pi}{2}\right]$. A $C_{0}$-semigroup $\{T(t)\}_{t \geq 0}$ on $X$ is said to be an analytic semigroup on $X$ if it has an extension $\{T(z)\}_{z \in \Delta_{\theta}}$ which is analytic in $\Delta_{\theta}$ and satisfies $\lim _{\Delta_{\delta} \ni z \rightarrow 0} T(z) x=x$ for $x \in X$ and $\delta \in(0, \theta)$. We say that $\{T(z)\}_{z \in \Delta_{\theta}}$ is bounded if there exists a constant $M>0$ such that $\|T(z)\| \leq M$ for $z \in \Delta_{\theta}$.

(b) Let $C$ be a bounded injective operator on $X$. A strongly continuous family $\{S(t)\}_{t \in \mathbf{R}}$ of bounded operators on $X$ is called a $C$-regularized group if $S(0)=C$ and $S(t) S(s)=S(t+s) C$ for $t, s \in \mathbf{R}$. Its generator is $A x=\left.C^{-1} \frac{d}{d t} S(t) x\right|_{t=0}$ with maximal domain.

We remark that if $A$ generates a bounded analytic semigroup $\{T(z)\}_{z \in \Delta_{\pi / 2}}$ on $X$, then by Theorem 17.9.1 and 17.9.2 in [13] $i A$ generates a bounded $C_{0}$-group $\{T(i s)\}_{s \in \mathbf{R}}$, where $T(i s) x=\lim _{t \downarrow 0} T(t+i s) x$ for $x \in X$. Furthermore, it is obvious that $A$ generates a bounded analytic semigroup on a Hilbert space if $A$ is self-adjoint and non-positive.

Let $n \in \mathbf{N}, m \in \mathbf{N} \backslash\{1\}$ and $c_{m n}=\int_{\mathbf{R}^{n}} \exp \left(-\frac{1}{4}|x|^{m /(m-1)}\right) d x$. For every $p \in[1, \infty)$, define the family $\left\{G_{p}(t)\right\}_{t \geq 0}$ of operators on $L^{p}\left(\mathbf{R}^{n}\right)$ by $G_{p}(0)=I$ and $G_{p}(t) f=k_{t} * f(t>0)$, where

$$
k_{t}(x)=\frac{1}{c_{m n} t^{n / m}} \exp \left\{-\frac{1}{4}\left(\frac{|x|^{m}}{t}\right)^{1 /(m-1)}\right\} \quad \text { for } x \in \mathbf{R}^{n} \text { and } t>0 .
$$

Then $\left\{G_{p}(t)\right\}_{t \geq 0}$ is strongly continuous and, by Young's inequality, $\|G(t)\|_{p, p} \leq$ $\left\|k_{t}\right\|_{1}=1$ for $t \geq 0$. In the case $m=2,\left\{G_{p}(t)\right\}_{t \geq 0}$ coincides with the Gaussian semigroup on $L^{p}\left(\mathbf{R}^{n}\right)$ with the Laplacian $\Delta_{p}$ as its generator.

The following definitions and results can be found in, e.g., [2, 12].

Definition 1.2. We say that a $C_{0}$-semigroup $\{T(t)\}_{t \geq 0}$ on $L^{p}(\boldsymbol{\Omega})$ has an (upper) Gaussian estimate of order $m$ if there exist constants $M, b>0$ such that

$$
|T(t) f| \leq M G_{p}(b t)|f| \text { for } t \geq 0 \text { and } f \in L^{p}(\boldsymbol{\Omega}) .
$$

If a $C_{0}$-semigroup $\{T(t)\}_{t \geq 0}$ on $L^{2}(\boldsymbol{\Omega})$ has a Gaussian estimate of order $m$, it follows from $\left\|G_{p}(t)\right\|_{p, p} \leq \overline{1}(t \geq 0)$ that $\|T(t) f\|_{p} \leq M\|f\|_{p}$ for $f \in L^{p}(\boldsymbol{\Omega}) \cap$ $L^{2}(\boldsymbol{\Omega})$. By the Riesz-Thorin interpolation theorem, there exists a family $\left\{T_{p}(t)\right\}_{t \geq 0}$ of bounded operators on $L^{p}(\boldsymbol{\Omega})$ such that $T_{p}(t) f=T_{2}(t) f \equiv T(t) f$ for $f \in L^{p}(\boldsymbol{\Omega}) \cap$ $L^{2}(\boldsymbol{\Omega})$. Then for every $p \in[1, \infty),\left\{T_{p}(t)\right\}_{t \geq 0}$ is a $C_{0}$-semigroup on $L^{p}(\boldsymbol{\Omega})$ and has 
a Gaussian estimate of order $m$. We call $\left\{T_{p}(t)\right\}_{t \geq 0}$ the consistent semigroup on $L^{p}(\boldsymbol{\Omega})$.

Definition 1.3. Let $1 \leq p, q \leq \infty$. $T \in B\left(L^{p}(\boldsymbol{\Omega}), L^{q}(\boldsymbol{\Omega})\right)$ is called an integral operator, if there exists a measurable function $k: \boldsymbol{\Omega} \times \boldsymbol{\Omega} \rightarrow \mathbf{C}$ such that for all $f \in L^{p}(\boldsymbol{\Omega}), k(x, \cdot) f(\cdot) \in L^{1}(\boldsymbol{\Omega}) x$-a.e. and $(T f)(x)=\int_{\boldsymbol{\Omega}} k(x, y) f(y) d y x$-a.e.. In this case, we say that $k$ is the kernel of $T$ and write $T \sim k$.

Lemma 1.4. (a) Let $1 \leq p, q \leq \infty$ and $T \in B\left(L^{p}(\boldsymbol{\Omega}), L^{q}(\boldsymbol{\Omega})\right)$ be an integral operator with kernel $k$. Suppose $T_{0} \in B\left(L^{p}(\boldsymbol{\Omega}), L^{q}(\boldsymbol{\Omega})\right)$ such that $\left|T_{0} f\right| \leq T|f|$ for $f \in L^{p}(\boldsymbol{\Omega})$. Then $T_{0}$ is an integral operator and $\left|k_{0}(x, y)\right| \leq k(x, y) x$,y-a.e., where $k_{0} \sim T_{0}$.

(b) The formula $\left(T_{k} f\right)(x)=\int_{\Omega} k(x, y) f(y) d y$ establishes an isometric isomorphism $k \mapsto T_{k}$ of $L^{\infty}(\boldsymbol{\Omega} \times \boldsymbol{\Omega})$ onto $B\left(L^{1}(\boldsymbol{\Omega}), L^{\infty}(\boldsymbol{\Omega})\right)$.

\section{Estimates OF ANALYTIC SEMigroups AND REgulARIZED GROUPS}

The first result is about the kernel estimate of analytic semigroups. In the sequel, we will denote by $M$ a general positive constant, and let $p \in[1, \infty)$.

Theorem 2.1. If a bounded analytic semigroup $\{T(z)\}_{z \in \Delta_{\pi / 2}}$ on $L^{2}(\boldsymbol{\Omega})$ has a Gaussian estimate of order $m$, then $T(z)$ is an integral operator for every $z \in \Delta_{\pi / 2}$, and there exists a constant $c>0$ such that

$$
|k(z, x, y)| \leq M(\operatorname{Re} z)^{-n / m} \exp \left\{-c \operatorname{Re} z(|x-y| /|z|)^{m /(m-1)}\right\}
$$

for $x, y \in \mathbf{\Omega}$ and $\operatorname{Re} z>0$, where $k(z, \cdot, \cdot) \sim T(z)$.

Proof. Since $\{T(t)\}_{t \geq 0}$ satisfies a Gaussian estimate, it follows from Lemma 1.4(a) that for every $t>0, T(t)$ is an integral operator and its kernel satisfies

$$
|k(t, x, y)| \leq M k_{b t}(x-y) \quad \text { for } x, y \in \mathbf{\Omega}
$$

where $k_{t}$ is given by (1.1). By duality and Hölder's inequality we have

$$
\|T(t)\|_{2,1}=\|T(t)\|_{\infty, 2} \leq M\left\|k_{b t}\right\|_{2} \leq M t^{-n /(2 m)} \text { for } t>0 .
$$

If $z=t+i s$ with $t>0$, then by the remark after Definition 1.1

$$
\|T(z)\|_{\infty, 1} \leq\|T(t / 2)\|_{\infty, 2}\|T(i s)\|_{2,2}\|T(t / 2)\|_{2,1} \leq M t^{-n / m} .
$$

Thus, from Lemma 1.4(b), the kernel of $T(z)$ exists and satisfies

$$
|k(z, x, y)| \leq M t^{-n / m} \quad \text { for } x, y \in \boldsymbol{\Omega} \text { and } \operatorname{Re} z>0 .
$$

Similarly to (2.3), we can show that $z \mapsto T(z)$ is analytic in norm $\|\cdot\|_{\infty, 1}$, and so $z \mapsto k(z, x, y)$ also is analytic for fixed $x, y \in \boldsymbol{\Omega}$. The estimate (2.1) now follows from (2.2), (2.4) and Lemma 9 in [6].

Now, we can show the estimate of analytic semigroups on the right halfplane.

Theorem 2.2. Let the conditions of Theorem 2.1 be satisfied. Then $\left\{T_{p}(t)\right\}_{t \geq 0}$ can be extended to an analytic semigroup $\left\{T_{p}(z)\right\}_{z \in \Delta_{\pi / 2}}$ on $L^{p}(\boldsymbol{\Omega})$ satisfying $T_{p}(z) \sim$ $k(z, \cdot, \cdot)$ and

$$
\left\|T_{p}(z)\right\|_{p, p} \leq M(|z| / \operatorname{Re} z)^{2 n\left|\frac{1}{2}-\frac{1}{p}\right|} \quad \text { for } \operatorname{Re} z>0
$$


Proof. Define

$$
\left(T_{p}(z) f\right)(x)=\int_{\boldsymbol{\Omega}} k(z, x, y) f(y) d y \quad \text { for } f \in L^{p}(\boldsymbol{\Omega}) \text { and } \operatorname{Re} z>0 .
$$

To observe that the family is well defined, let

$$
k_{0}(z, x)=\exp \left\{-c \operatorname{Re} z(|x| /|z|)^{m /(m-1)}\right\} \quad \text { for } x \in \mathbf{R}^{n} \text { and } \operatorname{Re} z>0 .
$$

Then by (2.1)

$$
\left|T_{p}(z) f(x)\right| \leq M(\operatorname{Re} z)^{-n / m} \int_{\mathbf{R}^{n}} k_{0}(z, x-y)|\widetilde{f}(y)| d y \quad \text { for } f \in L^{p}(\boldsymbol{\Omega})
$$

where $\widetilde{f}(y)=f(y)$ if $y \in \boldsymbol{\Omega}$ and 0 if not. It therefore follows from Young's inequality that

$$
\begin{aligned}
\left\|T_{p}(z)\right\|_{p, p} & \leq M(\operatorname{Re} z)^{-n / m} \int_{\mathbf{R}^{n}} k_{0}(z, x) d x \\
& =M(|z| / \operatorname{Re} z)^{n} \int_{\mathbf{R}^{n}} \exp \left\{-c|y|^{m /(m-1)}\right\} d y \\
& \leq M(|z| / \operatorname{Re} z)^{n} \quad \text { for } \operatorname{Re} z>0 .
\end{aligned}
$$

By the analyticity of $z \mapsto k(z, x, y)$ we can show that $\left\{T_{p}(z)\right\}_{z \in \Delta_{\pi / 2}}$ is an analytic semigroup on $L^{p}(\boldsymbol{\Omega})$ (cf. the proof of Theorem 2.4 in [17]). Let $1 \leq p \leq 2$. It follows from (2.6), our assumptions and the Riesz-Thorin interpolation theorem that

$$
\left\|T_{p}(z)\right\|_{p, p} \leq M\left\|T_{1}(z)\right\|_{1,1}^{\frac{2}{p}-1}\|T(z)\|_{2,2}^{2-\frac{2}{p}} \leq M(|z| / \operatorname{Re} z)^{2 n\left(\frac{1}{p}-\frac{1}{2}\right)}
$$

for $\operatorname{Re} z>0$. By duality (2.5) still holds for $2 \leq p<\infty$.

The conclusion that $\left\{T_{p}(t)\right\}_{t \geq 0}$ can be extended to an analytic semigroup $\left\{T_{p}(z)\right\}_{z \in \Delta_{\pi / 2}}$ on $L^{p}(\boldsymbol{\Omega})$ is due to Hieber [12, Theorem 2.3].

Combining Theorem 2.2 with [4, Theorem 2.1] we obtain the following result.

Theorem 2.3. Let the conditions of Theorem 2.1 be satisfied, and let $A_{p}$ be the generator of $\left\{T_{p}(t)\right\}_{t \geq 0}$. If $\alpha>2 n\left|\frac{1}{2}-\frac{1}{p}\right|$, then $i A_{p}$ generates a $\left(1-A_{p}\right)^{-\alpha_{-}}$ regularized group $\left\{S_{p}(t)\right\}_{t \geq 0}$ on $L^{p}(\boldsymbol{\Omega})$ satisfying

$$
\left\|S_{p}(t)\right\|_{p, p} \leq M\left(1+|t|^{2 n\left|\frac{1}{2}-\frac{1}{p}\right|}\right) \quad \text { for } t \in \mathbf{R} .
$$

\section{Estimates of Resolvents And the SPECTRUm OF GENERATORS}

Throughout this section, we assume that $A_{p}$ is the generator of $\left\{T_{p}(t)\right\}_{t \geq 0}$. We start with the kernel estimate of $R\left(\lambda, A_{p}\right)\left(\lambda \in \Delta_{\pi}\right)$.

Theorem 3.1. Let the conditions of Theorem 2.1 be satisfied. Then for every $\lambda \in \Delta_{\pi}, R\left(\lambda, A_{p}\right) \sim k_{R}(\lambda, \cdot, \cdot)$ where

$$
k_{R}(\lambda, x, y)=\int_{\Gamma} e^{-\lambda z} k(z, x, y) d z \quad \text { for } x, y \in \mathbf{\Omega}
$$

with $\Gamma=\left\{r e^{-i \theta / 2} ; r \geq 0\right\}$ and $\theta=\arg \lambda$. Moreover, there exists a constant $\gamma>0$ such that

(a) If $m>n$, then for $x, y \in \boldsymbol{\Omega}$ and $\lambda \in \Delta_{\pi}$,

$$
\left|k_{R}(\lambda, x, y)\right| \leq M|\lambda|^{\frac{n}{m}-\frac{1}{2}}(|\lambda|+\operatorname{Re} \lambda)^{-\frac{1}{2}} e^{-\alpha|x-y|}
$$

where $\alpha=\gamma|\lambda|^{\frac{1}{m}-\frac{1}{2}}(|\lambda|+\operatorname{Re} \lambda)^{\frac{1}{2}}$. 
(b) If $m=n$, then for $x, y \in \boldsymbol{\Omega}(x \neq y)$ and $\lambda \in \Delta_{\pi}$,

$$
\begin{aligned}
\left|k_{R}(\lambda, x, y)\right| \leq & M(1+\operatorname{Re} \lambda /|\lambda|)^{-1 / 2}(1+|\log | \lambda|| \\
& +|\log (|\lambda|+\operatorname{Re} \lambda)|+|\log | x-y||) e^{-\alpha|x-y|} .
\end{aligned}
$$

(c) If $m<n$, then for $x, y \in \Omega(x \neq y)$ and $\lambda \in \Delta_{\pi}$,

$$
\left|k_{R}(\lambda, x, y)\right| \leq M(1+\operatorname{Re} \lambda /|\lambda|)^{(m-n-1) / 2}|x-y|^{-(n-m)} e^{-\alpha|x-y|} .
$$

Proof. The following proof is a modification of the proof of Theorem 2.2 in [12]. By (3.1) and (2.1) we have

$$
\left|k_{R}(\lambda, x, y)\right| \leq M \int_{0}^{\infty} e^{-\delta|\lambda| r}(\delta r)^{-n / m} \exp \left\{-c \delta\left(\frac{|x-y|^{m}}{r}\right)^{\frac{1}{m-1}}\right\} d r
$$

where $\delta=\cos (\theta / 2)$. Also, we note the following fact:

$$
a r+b r^{-1 /(m-1)} \geq m a^{1 / m}(b /(m-1))^{1-1 / m} \text { for } a, b, r>0 .
$$

(a) Substituting $a=\delta|\lambda| / 2$ and $b=c \delta|x-y|^{m /(m-1)}$ in (3.6) and using (3.5) we obtain

$$
\begin{aligned}
\left|k_{R}(\lambda, x, y)\right| & \leq M \delta^{-n / m} e^{-\alpha|x-y|} \int_{0}^{\infty} r^{-n / m} e^{-\delta|\lambda| r / 2} d r \\
& \leq M \delta^{-1}|\lambda|^{-1+n / m} e^{-\alpha|x-y|} .
\end{aligned}
$$

(3.2) follows now from the fact $\delta=2^{-1 / 2}(1+\operatorname{Re} \lambda /|\lambda|)^{1 / 2}$.

(b) Set $s=|x-y|^{-m} r$ and $v=\left(|\lambda|^{1 / m}|x-y|\right)^{1-m}$. Then by (3.5) and (3.6) (with $a=\delta|\lambda||x-y|^{m} / 2, b=c \delta / 2$ ) we obtain

$$
\begin{aligned}
\left|k_{R}(\lambda, x, y)\right| \leq & M \delta^{-1} \int_{0}^{\infty} \frac{1}{s} \exp \left\{-\delta\left(|\lambda||x-y|^{m}+c s^{\frac{-1}{m-1}}\right)\right\} d s \\
\leq & M \delta^{-1} \exp \left\{-\frac{1}{2} c \delta v^{-\frac{1}{m-1}}\right\} \int_{0}^{v} \frac{1}{s} \exp \left\{-\frac{1}{2} c \delta s^{\frac{-1}{m-1}}\right\} d s \\
& +M \delta^{-1} e^{-\alpha|x-y|} \int_{v}^{\infty} \frac{1}{s} \exp \left\{-\frac{1}{2} \delta\left(|\lambda||x-y|^{m}+c s^{\frac{-1}{m-1}}\right)\right\} d s \\
\leq & M \delta^{-1} e^{-\alpha|x-y|}\left\{\int_{0}^{1} \frac{1}{s} \exp \left\{-\frac{1}{2} c \delta s^{\frac{-1}{m-1}}\right\} d s\right. \\
& \left.+\int_{1}^{\max (v, 1)} \frac{1}{s} d s+\int_{1}^{\infty} \frac{1}{s} e^{-\delta s / 2} d s\right\} \\
\leq & M \delta^{-1}(1-\log \delta+|\log v|) e^{-\alpha|x-y|}
\end{aligned}
$$

and therefore (3.3) follows from $\delta=2^{-1 / 2}(1+\operatorname{Re} \lambda /|\lambda|)^{1 / 2}$.

(c) It follows from (3.5) and (3.6) (with $a=\delta|\lambda|, b=\frac{1}{2} c \delta|x-y|^{m /(m-1)}$ ) that

$$
\begin{aligned}
\left|k_{R}(\lambda, x, y)\right| & \leq M e^{-\alpha|x-y|} \int_{0}^{\infty}(\delta r)^{-n / m} \exp \left\{-\frac{c \delta}{2}\left(\frac{|x-y|^{m}}{r}\right)^{\frac{1}{m-1}}\right\} d r \\
& \leq M \delta^{m-n-1}|x-y|^{m-n} e^{-\alpha|x-y|} .
\end{aligned}
$$

Hence (3.4) holds. 
Finally, we conclude by Young's inequality that $k_{R}(\lambda, x, \cdot) f(\cdot) \in L^{1}(\boldsymbol{\Omega})$ for $f \in$ $L^{p}(\boldsymbol{\Omega})$. Since we have (cf. [19, p.62])

$$
R\left(\lambda, A_{p}\right) f=\int_{\Gamma} e^{-\lambda z} T_{p}(z) f d z \quad \text { for } f \in L^{p}(\boldsymbol{\Omega}),
$$

the claim $R\left(\lambda, A_{p}\right) \sim k_{R}(\lambda, \cdot, \cdot)$ follows from $T_{p}(z) \sim k(z, \cdot, \cdot)$ and Fubini's theorem.

When $m=2$, Arendt [2] proved that $R\left(\lambda, A_{p}\right)$ is an integral operator by a different method.

Next, we give the $L^{p}$-estimate of the resolvent $R\left(\lambda, A_{p}\right)\left(\lambda \in \Delta_{\pi}\right)$.

Theorem 3.2. Let the conditions of Theorem 2.1 be satisfied. Then

$$
\left\|R\left(\lambda, A_{p}\right)\right\|_{p, p} \leq M|\lambda|^{n\left|\frac{1}{2}-\frac{1}{p}\right|-\frac{1}{2}}(|\lambda|+\operatorname{Re} \lambda)^{-n\left|\frac{1}{2}-\frac{1}{p}\right|-\frac{1}{2}} \quad \text { for } \lambda \in \Delta_{\pi} .
$$

Proof. Let $\theta=\arg \lambda$. Then by (3.7) and (2.5) we obtain that

$$
\begin{aligned}
\left\|R\left(\lambda, A_{p}\right)\right\|_{p, p} & \leq M \int_{0}^{\infty} e^{-|\lambda| r \cos (\theta / 2)}(\cos (\theta / 2))^{-2 n\left|\frac{1}{2}-\frac{1}{p}\right|} d r \\
& =M|\lambda|^{-1}(\cos (\theta / 2))^{-2 n\left|\frac{1}{2}-\frac{1}{p}\right|-1} \\
& =M|\lambda|^{n\left|\frac{1}{2}-\frac{1}{p}\right|-\frac{1}{2}}(|\lambda|+\operatorname{Re}(\lambda))^{-n\left|\frac{1}{2}-\frac{1}{p}\right|-\frac{1}{2}}
\end{aligned}
$$

as claimed.

Finally, as a direct consequence of Theorem 2.2 and [6, Theorem 3], we obtain the $p$-independence of $\sigma\left(A_{p}\right)$, which generalizes Corollary 4.3 in [2].

Theorem 3.3. Let the conditions of Theorem 2.1 be satisfied. Then the spectrum $\sigma\left(A_{p}\right)$ is independent of $p \in[1, \infty)$.

\section{Applications}

In this section, we will apply the results in sections 2 and 3 to some differential operators.

Example 4.1. Consider the Schrödinger operator $i(\Delta-V)$ where $V$ is real valued, $V_{+} \in L_{\text {loc }}^{1}\left(\mathbf{R}^{n}\right)$ and $V_{-} \in K^{n}$ (the Kato class). Let $A_{p}=\Delta-V$ with $\mathcal{D}\left(A_{p}\right)=$ $\left\{f \in L^{p}\left(\mathbf{R}^{n}\right) ; \Delta f \in L^{p}\left(\mathbf{R}^{n}\right\} \cap \mathcal{D}\left(V_{+}\right)\right.$, where $\mathcal{D}\left(V_{+}\right)$is the maximal domain of $V_{+}$ in $L^{p}\left(\mathbf{R}^{n}\right)$. Then $A_{2}$ is self-adjoint, and $\left\{e^{\left(A_{2}-\omega\right) t}\right\}_{t \geq 0}$ has a Gaussian estimate of order 2 for some constant $\omega \in \mathbf{R}$ [20, Proposition B.6.7]. Thus by our theorems

(a) $A_{p}$ generates an analytic semigroup $\left\{T_{p}(z)\right\}_{z \in \Delta_{\pi / 2}}$ on $L^{p}\left(\mathbf{R}^{n}\right)$ satisfying $\left\|T_{p}(z)\right\|_{p, p} \leq M e^{\omega \operatorname{Re} z}(|z| / \operatorname{Re} z)^{2 n\left|\frac{1}{2}-\frac{1}{p}\right|}$ for $\operatorname{Re} z>0$.

(b) For $\alpha>2 n\left|\frac{1}{p}-\frac{1}{2}\right|, i A_{p}$ generates a $\left(1+\omega-A_{p}\right)^{-\alpha}$-regularized group on $L^{p}\left(\mathbf{R}^{n}\right)$ satisfying $(2.7)$.

(c) The resolvent $R\left(\lambda+\omega, A_{p}\right)\left(\lambda \in \Delta_{\pi}\right)$ satisfies the estimate (3.8).

(d) The spectrum $\sigma\left(A_{p}\right)$ is independent of $p \in[1, \infty)$.

Here (a) and (d) are respectively due to [17] and [10]. (b) and (c) improve respectively Theorems 2.7 and 2.6 in [18]. We can choose other potentials, such as $V_{+} \in K^{n}, V_{-} \in L^{\infty}\left(\mathbf{R}^{n}\right)$. In this case, (b) is due to [4]. Moreover, when $V \equiv 0$, the index $\alpha$ in (b) can be improved (see $[8,11]$ and the references therein). 
Example 4.2. Let $D_{j}=\partial / \partial x_{j}(1 \leq j \leq n)$. Consider the symmetric sesquilinear form

$$
a(f, g)=\sum_{1 \leq j, k \leq n} \int_{\Omega} a_{j, k} D_{j} f \overline{D_{k} g} d x+\int_{\Omega} V f \bar{g} d x
$$

on $L^{2}(\boldsymbol{\Omega})$, where $a_{j, k}=\bar{a}_{k, j} \in L^{\infty}(\boldsymbol{\Omega}), 0 \leq V \in L_{\mathrm{loc}}^{1}(\boldsymbol{\Omega})$, and $\sum_{j, k=1}^{n} a_{j, k} \xi_{j} \xi_{k} \geq$ $\delta|\xi|^{2} x$-a.e. for some $\delta>0$ and all $\xi \in \mathbf{R}^{n}$. Let $\mathcal{D}(V)$ be the maximal domain of $V$, and let $\mathcal{D}(a)=H_{0}^{1}(\boldsymbol{\Omega}) \cap \mathcal{D}(V)$ (Dirichlet boundary condition) or $=H^{1}(\boldsymbol{\Omega}) \cap \mathcal{D}(V)$ (Neumann boundary condition, in this case $\boldsymbol{\Omega}$ has the extension property). If $-A$ is associated with $a, A$ is self-adjoint and $\left\{e^{t(A-\omega)}\right\}_{t \geq 0}$ has a Gaussian estimate of order 2 for some $\omega \in \mathbf{R}$ [5]. Hence the conclusions (a)-(d) in Example 4.1 still hold, in which (a) and (d) are essentially due to [17] and [2], respectively.

Example 4.3. Consider the symmetric operator

$$
A(x, D) f(x)=\sum_{|\mu|,|\nu| \leq m} D^{\mu}\left\{a_{\mu, \nu}(x) D^{\mu} f(x)\right\}
$$

where $a_{\mu, \nu}=\bar{a}_{\nu, \mu} \in W^{|\mu|, \infty}\left(\mathbf{R}^{n}\right)(|\mu|,|\nu| \leq m)$ and $a_{\mu, \nu}(|\mu|=|\nu|=m)$ are Hölder continuous, uniformly on $\mathbf{R}^{n}$. Assume that $A(x, D)$ is strongly elliptic, i.e., there exists $\delta>0$ such that

$$
\sum_{|\mu|=|\nu|=m}(-1)^{m} a_{\mu, \nu}(x) \xi^{\mu} \xi^{\nu} \geq \delta|\xi|^{2 m} \quad \text { for } x, \xi \in \mathbf{R}^{n} .
$$

Define $A_{p}:=A(\cdot, D)$ with $\mathcal{D}\left(A_{p}\right)=\left\{f \in L^{p}\left(\mathbf{R}^{n}\right) ; A(\cdot, D) f \in L^{p}\left(\mathbf{R}^{n}\right)\right.$ distributionally\}. Then the conditions of Theorem 2.1 are satisfied by $\left(e^{t\left(A_{2}-\omega\right)}\right)_{t \geq 0}$ for some $\omega \in \mathbf{R}$ [9], and so the conclusions (a)-(d) hold, in which (a) (besides the estimate) and (d) are essentially due to [15]. In particular, using a result of Amann $[1$, Theorem 12.1] and [7, Theorem 3.14] we obtain that the Cauchy problem

$$
\begin{cases}\frac{\partial}{\partial t} f(t, x)=i A(x, D) f(t, x) & \text { for } x \in \mathbf{R}^{n} \text { and } t>0, \\ f(0, x)=f_{0}(x) & \text { for } x \in \mathbf{R}^{n}\end{cases}
$$

has a unique solution $f \in C\left([0, \infty), W^{2 m, p}\left(\mathbf{R}^{n}\right)\right) \cap C^{1}\left([0, \infty), L^{p}\left(\mathbf{R}^{n}\right)\right)$ for every $f_{0} \in W^{\alpha, p}\left(\mathbf{R}^{n}\right)$, where $\alpha>2 m\left(2 n_{p}+1\right)$ and $1<p<\infty$.

If the coefficients only are bounded measurable, i.e. $a_{\mu, \nu}=\bar{a}_{\nu, \mu} \in L^{\infty}\left(\mathbf{R}^{n}\right)$ $(|\mu|,|\nu| \leq m)$, then Davies [6] showed that the conclusion (d) is true for $2 m>n$. In the case $2 m<n$, he proved that the spectrum $\sigma\left(A_{p}\right)$ only is independent of $p$ for $p$ in a certain interval around $p=2$. Moreover, in the case of constant coefficients, we refer to $[3,11,16]$ for some related results.

\section{REFERENCES}

1. H. Amann, Existence and regularity for semilinear parabolic evolution equations, Ann. Scuola Norm. Sup. Pisa Cl. Sci. (4) XI (1984), 593-676. MR 87h:34088

2. W. Arendt, Gaussian estimates and interpolation of the spectrum in $L^{p}$, Differential Integral Equations 7 (1994), 1151-1168. MR 95e: 47066

3. M. Balabane and H. Emamirad, $L^{p}$ estimates for Schrödinger evolution equations, Trans. Amer. Math. Soc. 118 (1985), 357-373. MR 87e:35050

4. K. Boyadzhiev and R. deLaubenfels, Boundary values of holomorphic semigroups, Proc. Amer. Math. Soc. 118 (1993), 113-118. MR 93f: 47043

5. E. B. Davies, Heat kernels and spectral theory, Cambridge Univ. Press, Cambridge, 1989. MR 90e: 35123 
6. E. B. Davies, Uniformly elliptic operators with measurable coefficients, J. Funct. Anal. 132 (1995), 141-169. MR 97a:47074

7. R. deLaubenfels, Existence families, functional calculi and evolution equations, Lecture Notes in Math. 1570, Springer-Verlag, Berlin, 1994. MR 96b:47047

8. O. El-Mennaoui and V. Keyantuo, On the Schrödinger equation in $L^{p}$ spaces, Math. Ann. 304 (1996), 293-302. MR 97g:47032

9. A. Friedman, Partial differential equations of parabolic type, Prentice Hall, New Jersey, 1964. MR 31:6062

10. R. Hempel and J. Voigt, The spectrum of a Schrödinger operator in $L^{p}\left(\mathbf{R}^{\nu}\right)$ is p-independent, Comm. Math. Phys. 104 (1986), 243-250. MR 87h:35247

11. M. Hieber, Integrated semigroups and differential operators in $L^{p}$ spaces, Math. Ann. 291 (1991), 1-16. MR 92g:47052

12. M. Hieber, Gaussian estimates and holomorphy of semigroups on $L^{p}$ spaces, J. London Math. Soc. 54 (1996), 148-160. MR 97d:47041

13. E. Hille and R. S. Phillips, Functional analysis and semigroups, Amer. Math. Soc., Providence, RI, 1957. MR 19:664d

14. L. Hörmander, Estimates for translation invariant operators in $L^{p}$ spaces, Acta Math. 104 (1960), 93-140. MR 22:12389

15. Yu. A. Kordyukov, $L^{p}$-theory of elliptic differential operators on manifolds of bounded geometry, Acta Appl. Math. 23 (1991), 223-260. MR 92f:58164

16. Y. Lei, W. Yi, and Q. Zheng, Semigroups of operators and polynomials of generators of bounded strongly continuous groups, Proc. London Math. Soc. 69 (1994), 144-170. MR 95f: 47062

17. E. M. Ouhabaz, Gaussian estimates and holomorphy of semigroups, Proc. Amer. Math. Soc. 123 (1995), 1465-1474. MR 95f:47068

18. M. M. H. Pang, Resolvent estimates Schrödinger operators in $L^{p}\left(\mathbf{R}^{n}\right)$ and the theory of exponentially bounded C-semigroups, Semigroup Forum 41 (1990), 97-114. MR 91m:35170

19. A. Pazy, Semigroups of linear operators and applications to partial differential equations, Springer-Verlag, New York, 1983. MR 85g:47061

20. B. Simon, Schrödinger semigroups, Bull. Amer. Math. Soc. 7 (1982), 447-526. MR 86b:81001a

Department of Mathematics, Huazhong University of Science and Technology, Wuhan 430074, People's Republic of China

E-mail address: qzheng@hust.edu.cn

Department of Mathematics, Hubei University, Wuhan 430062, People's Republic of China

E-mail address: zhangjz@hubu.edu.cn 